\title{
Téoros
}

Revue de recherche en tourisme

\section{Le musée comme élément du voyage : aperçu historique}

\section{Marie-Janou Lusignan}

Volume 11, numéro 2, juillet 1992

Quand les musées s’ouvrent au tourisme...

URI : https://id.erudit.org/iderudit/1078084ar

DOI : https://doi.org/10.7202/1078084ar

Aller au sommaire du numéro

Éditeur(s)

Université du Québec à Montréal

ISSN

0712-8657 (imprimé)

1923-2705 (numérique)

Découvrir la revue

Citer cet article

Lusignan, M.-J. (1992). Le musée comme élément du voyage : aperçu historique. Téoros, 11(2), 3-6. https://doi.org/10.7202/1078084ar d'utilisation que vous pouvez consulter en ligne.

https://apropos.erudit.org/fr/usagers/politique-dutilisation/ 


\title{
Le musée comme élément du voyage : aperçu historique
}

\author{
Marie-Janou Lusignan ${ }^{*}$
}

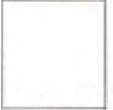

Choisissez au hasard n'importe quel guide touristique, consultezle, et vous constaterez assez rapidement qu'une large part de son contenu est consacré aux altraits culturels, et plus particulièrement aux institutions muséologiques. La fréquentation des musées parkaclientèle touristique estaujourd'hui d'une importancecapitaleet indéniable. Peutêtre croyez-vous que ce phénomène n'est imputablequ'ànotre histoirecontemporaine? Détrompez-wous. De tous temps, les voyageurs ont fréquenté les institutions muséologiques $^{(1)}$ lors de leurs scejours à l'étranger.

\section{De l'Antiquité à la Renaissance}

Déjả à l'époque de l' Antiquité, on se rendait principalement en Grèce, pour visiter les grands sanctuaires où des collections étaient formées pour rendre hommage aux divinités grecques et en Égypte, déjà fort populaire, pour ses nombreux monuments et sites célebres. Tous deux étaient le sujet exclusif de guides et d'études décrivant la beauté de leurs monuments et le prestige de leur passé.

Jusqu'à la Renaissance, trè̀s peu de gens se sont déplacés pour voyager et ce n'est qu'au XVII ${ }^{\circ}$ siecle qu' un renouveau se fit sentir. En effet, cette époque fut particulièrement marquée par ce qu'on appellerait aujourd' hui le tourisme artistique. L' Italie, la mère des arts, attirait alors erudits, nobles et rois qui se deplaçaient afin d'y admirer ses merveilles, généralement rassemblées dans de riches collections d'oeuvres d'art constituées par les grandes familles bourgeoises. Certains ramenaient chez soi de précieux souvenirs tels que toiles, statues, manuscrits, joyaux, et plusieurs artistes peintres, sculpteurs, orfevres, ciseleurs, menuisiers, musiciens, etc. étaient invités à venir pratiquer leur art à l'extêrieur de l'Italie.

C'est àcette époque aussi que naquit l'ancêtre des musées; le cabinet de curiosités, erésumé du monde, où prennent place des objets de la terre, des mers et des airs, ou des trois règnes, minéral, végétal et animal, à côté des productions de l'homme* ${ }^{(2)}$. Les collectionneurs parcouraient alors mers et monde pour acquérir ces objets de curiosité naturelle (naturalia)etartificielle(artificialia). Sans être ouverts au grand public, ces cabinets étaient fréquemment visités - sur rendez-vous - par les érudits et les illustres voyageurs de passage.

En Angleterre, c'est également au XVII siècle que s'inscrivit la tradition du Grand Tour. Le voyage à l'étranger était alors un moyen privilégié de compléter l'éducation des jeunes nobles anglais ${ }^{(3)}$. Ces derniers quittaient leur pays, accompagnés d'un tuteur, pour une durée de six mois à trois ans. Ils devaient effectuer des arrêts aux différents sites historiques, tels que Rome et Athènes. Les plus riches faisaient le Grand Tour de la France et de l'Italie et ramenaient à leur retour de nombreux tableaux et sculptures pour enrichir les collections de leurs familles.

\section{Le siècle des lumières}

Au XVIII" siecle, en Europe, se produisit une véritable révolution: les musées sont dorénavant accessibles au peuple. Au XVII ${ }^{e}$ siècle, seuls les voyageurs distingués, les savants et les intellectuels étrangers, avaient la possibilité d'admirer les collections privées. Par contre, au XVIII ${ }^{r}$ siècle, on admet peu à peu que l'accès au musće ne doit plus seulement être un privilège, mais un droit pour tous.

C'est au Louvre que ce renversement de mentalité s'est d'abord manifesté. Secoué par «' ellan révolutionnaire et les idéaux démocratiques du temps $w^{(4)}$, le Louvre a ouvert ses portes en 1793, dans le but de servir le peuple de la nouvelle République. Principal pôle d'attraction touristique parisien, ce musée était essentiellement visité par les étrangers, particulièrement par les Anglais. Ces derniers étaient très étonnés de voir que le musée étail envahi par le peuple. Il faut savoir cependant qu’à cette même époque, les collectionneurs Anglais étaient encore très fermés et très sélectifs quantau choix des visiteurs. Ils possédaient des collections excessivement riches (la tradition du Grand Tour s'étant perpétuée jusqu'au XVIII' siè- 
cle) et ils considerraient que leurs objets de collections, ayant une certaine valeur culturelle, ne pouvaient appartenir à la nation entière.

Avec cette récente ouverture au public du Palais du Louvre, il fallut prendre de nouvelles précautions afin d'éviter que les objets soient touchés, manipules ou volés. Pour eviter la détérioration des objets d'art, on émit, dès 1794 , des restrictions quant aux jours de visite des citoyens. Les touristes érangers étaient toutefois en tout temps les bienvenus car won se devait de leur offrir l'image de la richesse et de la puissance nationale ${ }^{(5)}$. Au fil des ans, les règlements devinrent de plus en plus sévères. Bientôt on ne retrouva dans les salles du Louvre, que les touristes provinciaux et étrangers, ainsi que la bourgeoisic parisienne qui avaient pris l'habitude d'aller y faire un tour.

A partir de la deuxième moitié du $\mathrm{XIX}^{c}$ siecle, la visite du Louvre devint une obligation pour tous les étrangers et les provinciaux. Les guides touristiques de l'époque présentaient ale Musée du Louvre comme l'un des monuments a voir absolument au même titre que le Jardin des Plantes et la Sainte-Chapellew. Peu de ces touristes étaient des connaisseurs en matière d'art, et la plupart d'entre eux parcouraient les salles les yeux rivés dans leur guide, à la recherche exclusive des chefs-d'oeuvre. Pour eux, le Louvre se réduisait wà une dizaine d'oeuvres - sorte de musce imaginaire du parfait touriste - ... $y^{(T)}$ Les choses ont-elles vraiment changé aujourd'hui?

\section{L'ère de la révolution industrielle ou le siècle du tourisme}

Le XIX ${ }^{e}$ siecle bouleversa le monde: les découvertes technologiques et scientifiques, l'introduction d'une notion nouvelle, celle de temps libre (c'est à partir de cette époque que l'on peut donc réellement parler de tourisme) changèrent radicalement les modes de vie. La conception du moteur à vapeur, et par la suite, celle de la locomotive, est sans conteste l' invention qui aura eu le plus d'impact. Le développement des réseaux de chemins de fers et des autres moyens de transport incitèrent des promoteurs à developper l'industrie du voyage. Thomas Cook fonda, en 1841, la première agence de voyages au monde. II organisa des voyages vers Londres, Dublin et Paris pour visiter... les grandes Expositions Universelles. Elles attiraient un très grand nombre de visiteurs, essentiellement des touristes étrangers: pour l'Exposition Universelle de Paris en 1889. Thomas Cook publia dix millions d'imprimés. Ces expositions sont a l'origine des musces modernes, c'est-ă-dire les musees qui, en terme de présentation d'objets, prennent en considération la vie sociale de la communauté environnante. Elles permirent également la création de nouveaux musées puisqu'à la clôture d'une Exposition, certains pavillons étaient conservés puis convertis en musées. Les Expositions Universelles initièrent alors une véritable révolution au sein des musées. Grâce à elles, les musćes héritèrent du pouvoir, de l' influence sociale qu'ils n'avaient pu acquérir auparavant.

En Amérique du Nord, les musées se multiplient. Les Etats-Unisassistenta lanaissance d'institutions majeures telles le MetropolitanMuseum of ArtdeNew YorketleMuseum of Fine Arts de Boston qui, pour constituer leurs collections d'antiquites, effectuaient des expéditions en Égypte, en Grèce et à l'Ile de Chypre.

Au Québec, comme ailleurs dans le monde, wa période qui s'étend de 1870 à 1900 est considérée comme l'age d'or des musées d'histoire naturelle...p (o). Plusieurs musées vont alors naître au Québec, et parmi les premiers à être fondés a Montréal, on peut compter le Musée Italien (ou le Musée Del Vecchio), et le Natural History Society of Montreal (on peut retrouver aujourd" hui une partie de la collection de ce demier au musée Redpath de l'Université McGill).

Plus tard, en 1842, la Commission géologique du Canada füt mise sur pied par le gouvemement du Canada-Uni. C'esten 1851 que la Commission présenta sa collection pour la première fois à l'étranger, à la première Exposition Universelle qui se tenait alors à Londres. Son succes fut si grand que la Commission géologique eut la responsabilité de représenter le Canada lors des Expositions Universelles subséquentes.

Malgre l'importance de toutes ces institutions, les manifestations de type muséologique les plus populaires à Montréal à cette époque étaient les expositions itinérantes (environ une cinquantaine pour les années 1817-1847). Essentiellement étrangères, elles se tenaient principalement durant la période estivale dans ales hôtels, auberges et cafés de la ville, dont les propriétaires voyaient évidemment là une occasion d'accroitre leur clientèles ${ }^{(9)}$. Ces expositions etaient majoritairement de type historique, artistique, zoologique, maiselles présentaient aussi des curiosites humaines et animales, comme le faisaient les cabinets de curiosités européens des XVI ${ }^{e}$ et XVII ${ }^{e}$ siècle. Leur fréquentation était un usigne de distinction sociale, preuve que l'on a du goût, de l'education et des loisirs, en même temps qu'une position à tenir et peut-être aussi quelqu'anbition à satisfaire ${ }^{(10)}$. N'est-ce pas là aussi, la description du parfait touriste?

En 1850, il y eut à Montréal, une exposition industrielle et commerciale.Son mandat était d'effectuer la présélection des exposants de la première Exposition Universelle de Londres en 1851. A cette occasion, des promoteurs ont offert aux gens de la ville deQuébec, qui désiraient participer à cette exposition, des forfaits voyages comprenant le transport, l'hébergement à bord de bateaux à vapeur et les repas. Un très grand nombre d'Américains sont aussi venus à Montréal à cette occasion.

À Québec, le sculpteur et doreur Pierre Chasseur fonda un musce d'histoire naturelle en 1826. Il obtint quelques subventions de l'État pour effectuer des voyages de collectionnement. Dix ans plus tard, sa collection fut rachetée par le Gouvernement du Québec, lui donnant ainsi le statut de premier musée public au Canada. Chasseur, ayant pris part aux Événements de 1837 et 1838 , futarrêté et le gouvernement céda sa collection à la Literary and Historical Society of Quebec (LHSQ). Malheureusement, la LHSQ fut complètement anéantie par le feu en $\mathbf{1 8 6 2 .}$

Les institutions d'enseignement, elles aussi, se doterent de collections d'histoire naturelle dans le but de parfaire et de compléter la formation académique de leurs êlèves. Afin d'enrichir leurs collections, certains établissements financèrent des expéditions au Québecet aux États-Unis; l'Université Laval commandita en 1872 un voyage en Floride. Certains de ces muś́es acquirent une réputation internationale: le Musée de l'Université Laval, par exemple, se fit connaître au Canada et à l'étranger en favorisant des échanges avec le Musée de la Commission géologique (relocalisé a Ottawa depuis 1881), le Smithsonian Institute de Washington, le Museum d'histoire naturelle de Paris et le Musce royal d" histoire naturelle de Bruxelles.

Au Québec, cependant, on ne se passionnait pas uniquement pour les spécimens d'ordre naturels. Dans son livre de 1873 intitule L'album du touriste, J.-M. LeMoine décrit 
avec minutie dans l'un de ses chapitres, les richesses artistiques de la ville de Québec: «Il y a dans les églises de Québec, des toiles et des peintures d'un grand prix. Les écoles françaises et flamandes y sont présentees avec avantage. Plusieurs des originaux sont l'oeuvre de grands maîtres... il en est un bon nombre qui font chaque anné, l'admiration des connaisseurs et des touristes.... ${ }^{(11)}$ Les églises jouaient en quelque sorte à cette époque, le rôle des musées tels que connus aujourd'hui, en assurant la conservation d'oeuvres d'art.

\section{Le siècle des loisirs}

Au XX' siecle, le tourisme s'organise de plus en plus: en Europe, toutes sortes de clubs, d'organismes etd'associations sont crés.s. Le perfectionnement des moyens de transport, l'augmentationdesrevenus ${ }^{(12)}$, et le droit aux congés payés ont génêreusement contribué à

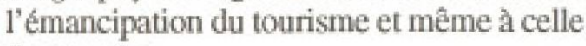
des musées.

Au début du siécle, les pares nationaux, les sociétés historiques et maisons historiques américaines durent leur succès à l'invention de l'automobile. S'adressant essentiellement à une clientèle familiale, ces derniers voyageaient par le moyen de transport le plus économique: la voiture. Puis, le développement de l'aviation civile apress la Deuxième Guerre mondiale fit surgir un nouveau phenomène: le tourisme de masse; le tourisme est maintenant collectivement un besoin primaire. On voit aussi apparaître en Europe, comme au Qub́bec, de nouvelles catégories de musées: écomusée, musée de plein-air, muséc de voisinage, musée des sciences et techniques, musée des arts et traditions populaires, etc.

De tout temps, les musces ont accueilli des visiteurs provenant de régions ou de pays lointains. Au XXe siècle, cette réalité subsiste toujours et atteint des dimensions presque démesurées. Donnons deux exemples, l'un s'appliquantà laFrance et l'autreà la Hongrie.

Il est impressionnant de constater à quel point les institutions muséales françaises subissent, durant la perriode estivale, une forte croissance de leur taux de fréquentation: certains musées atteignent des taux records allant au-delà des $7000 \%$ d'augmentation! Cet engouement pour les musées se fait surtout sentir dans les régions les plus réputées au point de vue touristique et coincide avec les mois de la haute saison. Pendant le reste de l'année, le nombre d'entrées baisse radicalement. C'est donc dire que l'essentiel de leur clientèle est composce de voyageurs. \&La relation étroite entre le tourisme... et la fréquentation des musées du point de vue de l'augmentation des entrées est démontrếe par des chiffres révélateurs. Si l'on compare les chiffres dans le domaine du tourisme international avec ceux de la fréquentation des musées dans le pays, on est surpris par l'analogie du tracé des diagrammes ${ }^{(13)}$.

Durant la saison touristique, $85 \%$ des visiteurs du Château de Versailles, et $70 \%$ des visiteurs des musées français, proviennent de l'étranger. En plus de cette augmentation saisonnière, on estime en France que durant les 25 dernières années, la fréquentation des monuments a doublé et que celle des musées a quadruplé.

Ce phénomène est identique dans les pays de l'Est, comme par exemple la Hongrie: en 1971,7500000 personnes avaient visite leurs musées et ce chiffre s"élevait en 1982 a 17700000 . aCette augmentation spectaculaire peut être vue comme étant en étroite relation avec trois tendances nationales qui ont surgi simultanément: une augmentation de l'intérêt général du public s'est manifestếe pour les sujets culturels, artistiques et historiques; au niveau de la culture et de l'éducation, lapolitique officielle a, elle aussi, accordé une attention plus grande aux moyens de faire connaîtreet de présenter les richesses des musées; et, finalement, l'augmentation du tourismeapparaît comme une conséquence directe de l'accroissement du temps de loisir. Ce phénomène de l'intensification du tourisme intemational a eu en Hongrie des résultats remarquables s ${ }^{(15)}$. De manière générale, ces tendances s'étendent à l'ensemble du monde occidental. À ces explications peut s'ajouter une autre justification: la démocratisation des musées. Ces demiers ont souhaité la présence dans leurs institutions d'un public plus diversifié et par conséquent, ont favorisé leur accessibilité par le développement de moyens d'accueil et d'activités pédagogiques.

Le tourisme peut-il contribuer, ou contribuet-ill vraiment au développement des musées? Oui, si dans un premier temps, on considere les données statistiques présentées plus haut, qui décrivent de manière absolue l'étroite relation qui existe entre les musées et le tourisme, et dans un deuxième temps, les études qui révèlent l'accroissement annuel du mouvement migratoire international des voyageurs. Ayant à transiger avec un nou-

\section{Tableau 1}

Fréquentation de quelques sites culturels et touristiques en France en 1989(14)

Site

Nombre de visiteurs/an

Musée du Louvre

Centre Pompidou

4100000

Château de Versailles

7112000

Notre-Dame de Paris

2160000

8000000

veau public, public dont les caractéristiques se modifient continuellement selon le pays d'origine, les musées doivent alors s'ajuster constamment aux exigences de ces visiteurs, même s'ils ne constituent pas leur public cible. Dans un deuxième temps, si on considère l'impact, généralement négatif, du tourisme de masse sur la preservation des biens culturels (vandalisme, vol, détérioration, conséquence de la surfréquentation, etc.), les musées doivent trouver des solutions à ces nouveaux problèmes. Toutes ces adaptations côtoient inévitablement le processus de développement, d'évolution des musées.

Mais inversement, pouvons-nous dire que lesmusóesdéterminentetenrichissentl'image de marque d'une destination touristique? Contribuent-ils à l'essor du tourisme régional ou urbain? "Considêré comme élément complémentaire, le musée est une composante d'un produit touristique au même titre que l'ensemble des autres activités culturelles, sportives, récréatives... Mais cet équipement s'avère essentiel dans le cas d'une destination touristique qui recherche la notoriété... ainsi la présence d'un musée de dimension respectable et d'un contenu varié situe la destination dans une classe supérieure et ajoute a sa renommée. En d'autres termes, une destination touristique est vraiment complete lorsque l'art et la culture atteignent un certain degré de qualité et occupent un espace minimal. (i6) $^{(16}$

En 1991, parmi les dix attractions touristiques les plus fréquentées a Montréal, on retrouve majoritairement des institutions de type muséologique: l'Insectarium, le Jardin Botanique, I'Aquarium, le Musée des BeauxArts, le Planétarium Dow, le Musée David M. Stewart et le Château Ramesay. 


\section{Conclusion}

Les musces et le tourisme ne peuvent vivre l'un sans l'autre; ils coexistent de manière semi-indépendante ă l'intérieur d'un même ensemble. La preuve en est faite lorsque nous consultons les guides touristiques, ou tous les ouvrages faisant la description de destinations touristiques particulières: ils possedent tous un contenuculturel qui traite, entre autre chose, des musées. Plus un guide touristique recommandera la visite d'un musće et plus de publicite sera faite autour d'un musce, plusilserasusceptibled'être populaireauprès des touristes. Les conséquences de cette popularité peut, dans certains cas, être désastreuse sur le plan de la conservation. Cependant, la mise en valeur du patrimoine à des fins touristiques n'a pas que des effets négatifs; elle peut également contribuer de manière fort positive a la sauvegarde de notre patrimoine culturel, tangible et intangible. aCette relation entre le tourisme et la culture conduit non pas à une dégénérescence des arts mais contribue dans bien des cas à leur conservation et à leur survie. Il est courant que le tourisme donne un elan a la revitalisation de métiers artisanaux, des arts d'expression corporelle comme le folklore, a une architecture typique et à la création $\mathrm{d}^{7}$ Équipements muséologiques de diverses catégoriescomme le musće d'art, l'écomusce et même l'économusée dans lequel la rentabilité joue un rôle fondamental. $\AA$ ce titre, le muséeest en quelque sorte une vitrine permanente autant pour l'histoire, l'art, la science, en offrant aux touristes comme aux gens du milieu, une attraction complémentaire à une destination de voyage d"agrément. $D^{\prime}$ autant plus que dans de nombreux sites, le musée constitue l'unique lieu où le touriste peut retrouver les vestiges d'une époque révolue, alors qu'adisparu toute trace historique et patrimoniale pour laisser place au modernisme. En somme, le touriste apprécie une destination qui lui permet de changer de paysage momentanément, ce contraste se manifestant par un milieu qui présente une physionomiedifférente de celle qui lui est familière. De tous les facteurs répondant le mieux à cette attente, les plus importants sont ceux se rapportant au domaine socio-culturel, à la culture. Cela est d'autant plus vrai que lacultureet chacune de ses composantes décrivent d'une manière vivante le dynamisme évolutif des habitants; ce que ne peuvent exprimer les attraits géographiques et physiques du milieu, ceux-ci etant statiques, ${ }^{(17)} \underset{f}{ }$

\section{RÉFÉRENCES}

(1) Outre les musées désignés comme tel, sont appeles ainstitution muselogiquen, les sites ef monuments historiques, archélogiques. ethnographiques et naturels: les jardins botaniques, zoologiques, aquariums, etc; les parcs naturels: les centres scientifiques et planétariums: les institutions de conservation et les galeries d'expositions.

(2) Antoine Schnapper, Le gdant, la licorne ot la tulipe, p. 9.

(3) L'expression Grand Tour apparut d'aborden France pour designer le voyage culturel par excellence, mais les Anglais se l'approprierent aussitot pour indiquer la consecration d'une education aristocratique. The Oxford English Dictionary deffinit in great tour (great circuit) comme étant sa tour of the princpal cities and places of interest in Europe; formely supposed to be an essential part of the education of young men of good birth or fortune. To make the grand tour : (Giuseppe Loy Puddu, Geographie touristique: communications ot transports, p. 22.

(4) Chantale Georgell, "Les premiers visiteurs du Louvres L'Histoire, no 130, févier 1990, p. 30.

(5) Idem, p. 30 .

(6) Idem, p. 32 .

(7) Idem, p. 33.

(8) Paul Carle et Raymond Duchesne, s'ordre des choses: cabinets et musees d'histoire naturelle au Québec (1824-1900) * Revue d'histoire de rAmbrique française, vol. 44, no 1, ete 1990, pp. 4.5.

(9) Hervó Gagnon, "Expositions et curiositésis, Mustes, wol. 13, no 2, juin 1991, p. 29.

(10) Paul Carle et Faymond Duchesne, op. cit, p. 5.

(11) Sir James Macpherson Le Moine, L'abum du touriste, $\mathrm{p}, 19$.

(12) Le taylorisme qui apparaít au début du siècle. entraine un accroissement de la productivité qui va permettre a la société de s'enrichir de plus en plus en trava lant de moins en moins. Le syndicalisme, les luttes ouvrieres, les pressions sociales vont déclencher ce processus et faire en sorte qu'il se poursuive. En une cinquantaine d'année. les pays les plus industrialises ont vule nombre de personnes pouvant disposer d'assez de temps et d'argent pour s'offrir des loisirs augmenter de façon considérable.

(13) István Berta, r Tourime et muséew, Museum, vol. XOOV, no 4,1983 , p. 245.

(14) Extrait d un tableaude Sophie Élias, - Tourismeou patrimoine: faut-il choisir?.., Espace, no 104, juinjuillet $1990, \mathrm{p} .6$.

(15) Istuán Berta, aTourisme et museen, op cit. p. 244.

(16) Jacques Demers, "Le musete peut-il aider la cause touristique? : Musúes, vol, 13, no 1, mars 1991, pp. 7-8.

(17) Idem, pp. 6-7. 